\title{
Refinement Issues in Stochastic Multistage Linear Programming*
}

\author{
Karl Frauendorfer \\ Christina Marohn \\ University of St. Gallen \\ Institute of Operations Research \\ Holzstrasse 15 \\ 9010 St. Gallen
}

October 29, 1997

\begin{abstract}
Linear stochastic multistage programs are considered with uncertain data evolving as a multidimensional discrete-time stochastic process. The associated conditional probability measures are supposed to depend linearly on the past. This ensures convexity of the problem and allows application of barycentric scenario trees. These approximate the discrete-time stochastic process, and provide inner and outer approximation of the value functions.

The main issue is to refine the discretization of the stochastic process efficiently, using the nested optimization and integration of the dynamic, implicitely given value functions. We analyze and illustrate how errors evolve across nodes of the scenario trees.
\end{abstract}

${ }^{*}$ Research of this report was supported by Schweizerischer Nationalfonds Grant Nr. 21$39^{\prime} 575.93$ 


\section{Introduction}

In various applications decision makers are required to take uncertain future into account. In particular, today's decisions have to be taken without knowing prices or resources.

Let the uncertain evolvement of data over a finite planning horizon $[0, T]$ be described as a multidimensional discrete-time stochastic process $\left(\omega_{t}, t=0, \ldots, T\right)$ on a common Borel space $\left(\Omega, \mathcal{B}^{M}\right)$ with $\Omega \subset \mathbb{R}^{M}$ compact. In many problem statements both prices and demand or supply of resources are stochastic and induce specific structural properties of the value functions involved. This motivates us to decompose the multidimensional discrete-time stochastic process $\left(\omega_{t}, t=0,1, \ldots, T\right)$ into two stochastic parts, one referring to prices $\left(\eta_{t}, t=0,1, \ldots, T\right)$, the second $\left(\xi_{t}, t=0,1, \ldots, T\right)$ to demand or supply of resources, i.e. $\omega_{t}=\left(\eta_{t}, \xi_{t}\right)$.

Let $P$ represent the (regular) joint probability distribution of $\omega:=\left(\omega_{0}, \ldots, \omega_{T}\right)$. The associated regular conditional distributions with respect to $\omega_{t}$ are denoted $P_{t}\left(\cdot \mid \omega_{t-1}\right)$ for $t=1, \ldots, T$. All these have compact support. The time points $t=$ $0,1, \ldots, T$ at which decisions $u_{t} \in \mathbb{R}^{n}$ are taken, are supposed to be predefined. Setting $\eta:=\left(\eta_{0}, \ldots, \eta_{T}\right)$ and $\xi:=\left(\xi_{0}, \ldots, \xi_{T}\right)$, a corresponding mathematical program is written formally as

$$
\begin{array}{ll}
\min & \int_{\Omega}\left[\sum_{t=0}^{T} \rho_{t}\left(u_{t}, \eta_{t}\right)\right] d P(\eta, \xi) \\
\text { s.t. } & f_{t}\left(u_{t-1}, u_{t}\right) \leq h_{t}\left(\xi_{t}\right), \quad t=0,1, \ldots, T .
\end{array}
$$

The convention is that negative subscripts of variables indicate decisions of the past, negative subscripts of the stochastic data indicate data of the past, both of which represent the input data at the present stage $t=0$; in particular, stochastic data with subscript 0 are currently observed data and, hence, deterministic.

In (1), the cost $\rho_{t}(\cdot)$ at $t$ are determined by observation $\eta_{t}$ and decision $u_{t}$. The feasibility region for $u_{t} \in \mathbb{R}^{n}$ is supposed to depend on $u_{t-1}$ and on the observed outcome $\xi_{t} ; f_{t}\left(u_{t-1}, \cdot\right)$ is a vector-valued function in $u_{t}$ and represents the demand for resources, the components of $h_{t}\left(\xi_{t}\right)$ are the supply components of the resources at $t$. Decisions $u_{t}$ have to be selected at $t$ after $\left(\eta_{t}, \xi_{t}\right)$ is observed, but prior to the observations $\left(\eta_{t+1}, \ldots, \eta_{T}\right)$ and $\left(\xi_{t+1}, \ldots, \xi_{T}\right)$. According to this rule, nonanticipative or measurable decisions have to be determined, which minimizes the expected value of the overall cost and which satisfies the constraints.

The feasibility set, viewed as a constraint multifunction in $\xi$, is supposed to be strictly nonanticipative and convex compact-valued with a nonempty interior for every $\xi$. This ensures that for any nonanticipative and feasible decision $u_{t}$ there exist interior feasible and nonanticipative decisions $u_{t+1}, \ldots, u_{T}$ for any 
sequence of outcomes $\left(\xi_{t+1}, \ldots, \xi_{T}\right)$ (see Rockafellar and Wets 1976/1978 [45],[46] and Frauendorfer 1996 [23]).

Standard dynamic programming arguments yield optimal value functions $\phi_{t}(\cdot)$ corresponding to periods $t=0, \ldots, T$ (see e.g. Bertsekas 1995 [2], [3]). This allows to write the stochastic multistage programm as a sequence of nested twostage programs. Start at period $T$ with $\phi_{T+1}(\cdot):=0$ and define backwards for $t=T, \ldots, 0$ :

$$
\begin{aligned}
& \phi_{t}\left(u_{t-1}, \eta_{t}, \xi_{t}\right):=\min \rho_{t}\left(u_{t}, \eta_{t}\right)+\int_{\Omega_{t+1}} \phi_{t+1}\left(u_{t}, \omega_{t+1}\right) d P_{t+1}\left(\omega_{t+1} \mid \eta_{t}, \xi_{t}\right) \\
& \text { s.t. } \quad f_{t}\left(u_{t-1}, u_{t}\right) \leq h_{t}\left(\xi_{t}\right) .
\end{aligned}
$$

In case $\rho_{t}\left(u_{t}, \eta_{t}\right)$ are continuous convex-concave saddle functions, $f_{t}(\cdot)$ is convex vector-valued, $h_{t}(\cdot)$ is linear-affine in $\xi_{t}$, and in case the conditional probability distributions $P_{t+1}\left(\cdot \mid \eta_{t}, \xi_{t}\right)$ depend linearly on $\left(\eta_{t}, \xi_{t}\right)$ and are unaffected by the decisions taken, then it has been proven that for $t=1, \ldots, T$ the value functions $\phi_{t}\left(u_{t-1}, \eta_{t}, \xi_{t}\right)$ are continuous saddle functions, convex in $\left(u_{t-1}, \xi_{t}\right)$ and concave in $\eta_{t}$ with respect to their domains. Under these conditions, referred to as the convex case below, primal and dual solvability of the convex programs is ensured in (2). For details it is referred to Rockafellar and Wets 1976 [45], Frauendorfer 1994/1996 [22] and [23].

For the ease of exposition it will be helpful to use for (2) the notation

$$
\phi_{t}\left(u_{t-1}, \eta_{t}, \xi_{t}\right):=\min \left[\rho_{t}+\mathrm{E}_{t} \phi_{t+1}\right]\left(u_{t}, \eta_{t}, \xi_{t}\right) .
$$

One major challenge with (1) is the nested minimizations and multidimensional integrations of implicitely given value functions. Unlike many control type formulations, no analytical expressions can be expected within the stochastic multistage setting due to the non-smoothness of the value functions. For overcoming these difficulties numerically in the convex case we discretize the stochastic processes with respect to their outcomes taking into account the subdifferentiability of the value functions.

Let the conditional probability measures $P_{t}\left(\cdot \mid \omega_{t-1}\right)$ be successively discretized for $t=0, \cdots, T$, yielding discrete conditional probability measures $Q_{t}\left(\cdot \mid \omega_{t-1}\right)$ with corresponding support $A_{t}\left(\omega_{t-1}\right)$. This way, a scenario tree $A$ and the associated path probabilities $Q(\omega)$ are given according to

$$
\begin{aligned}
& A:=\left\{\omega \in \Omega \mid \omega_{t} \in A_{t}\left(\omega_{t-1}\right), \forall t \geq 1\right\} \\
& Q(\omega):=\prod_{t=1}^{T} Q_{t}\left(\omega_{t} \mid \omega_{t-1}\right) .
\end{aligned}
$$

Let the projections of $A$ onto $[0, t]$ be denoted $A^{t}$. Clearly, any stochastic process, which is discrete in both time and state, is representable as scenario tree. 
It is easily seen when the conditional probability measure $P_{t+1}\left(\omega_{t+1} \mid \eta_{t}, \xi_{t}\right)$ is discrete with finite support, that the stochastic two-stage programs in (2) has block structure. Then (1) may be written as a mathematical program with a dynamic block structure and high sparsity, whose size depends on the number of scenarios within the tree. This indicates why the solvability of stochastic multistage programs strongly benefits from sophisticated algorithms. Recent works include Rockafellar and Wets 1991 [47], Wets 1989 [51], Gassmann 1990 [27], Birge 1985/1994/1995 [5],[7],[6], Mulvey and Ruszczyński 1995 [38], Mulvey, Vanderbei and Zenios 1995 [40], Zenios 1991 [52], Robinson 1991 [44], Ruszczyński 1993 [49],[48], Dantzig 1990/1993 [13], [14], Edirisinghe and Ziemba 1994/96 [18], [17], [19], Kall, Ruszczyński and Frauendorfer 1988 [32], Infanger 1992/1994 [30],[31], Ermoliev and Wets 1988 [20], Kall and Wallace 1994 [34], Kall and Stoyan 1982 [33], Wets 1989 [51], Hiller and Eckstein 1994 [29].

However, one has to be aware of the fact that discretizing the conditional probability measures is a simplification of the real dynamics, which may have severe impact on the goodness of the surrogate problem. It is not difficult to define conditions based on which the discretization has to be refined, to ensure weak convergence of the discrete measures to the real probability measure and, hence, epi-convergence of the minimizers. But, due to the fact that the number of scenarios grows exponentially, the quality of discretizations has to be monitored carefully. For example, applying Monte-Carlo simulation to the conditional probability measures with a sample size of $10^{5}$ in each of 6 stages results in a scenario tree with $10^{30}$ possible scenarios. Suffering from the curse of dimensionality the corresponding deterministic equivalent program is numerically unsolvable. In the convex case, barycentric approximation allows design distinguished scenario trees that provide information on how accurate the real dynamics are approximated.

We discuss issues on how to refine the scenario trees taking into account the nested optimization and integration of the value functions. Hence, this work provides improvements within algorithmic procedures that solve stochastic multistage linear programs.

The paper is organized as follows: Section 2 introduces a multistage finance problem and releases its structural properties, which may be exploited within the refinement process. Section 3 reviews briefly barycentric scenario trees and the approximation of the value functions which help overcome the difficulties with nested minimizations and multidimensional integrations. Section 4 represents the major part of this work and investigates how the error evolves along the scenario tree backwards in time, and measures to what extent error arises from integration. Section 5 concludes. 


\section{Application in banking}

An example from banking is given to illustrate the application of multistage stochastic linear programming. Besides linearity, further structural properties are inherent in many financial problem statements and, hence, facilitate their solvability. Herein, we consider funding of non-fixed rate mortgages which face interest rate and prepayment risk. This constitutes an important problem for corporate financial managers of Swiss banks due to the risks these managers are exposed to.

Non-fixed rate mortgages offer the clients to finance their mortgages at current market rates which strongly correlate with the current interest rate level. It should be noted that the national bank not only monitors the mortgage rate but also defines some cap in case the market interest rates are too high. This is intended to protect the clients and, on one hand, reduces the credit risk for the bank, on the other hand, additional risk is faced with respect to the funding mechanism. In Switzerland, it happened over a rather long period within the last decade that the mortgage rate the clients had to pay was even less than the one-year-borrowing rate at which the bank funded a considerable part of their business. In such an interest rate environment the clients clearly prefer the variable rate mortgages. This increases the volume and the funding costs and therefore the risk the bank is exposed to. If the interest rate level is low, it has been observed that the mortgage rate is kept above a certain floor, causing fixedrate mortgages to fall even below the non-fixed ones. As a natural consequence, clients change their liabilities to the fixed-rate mortgages, causing the non-fixed rate volume to decrease in an environment where banks would have the possibility to fund their non-fixed rate business at low costs. This type of risk is known as prepayment risk. Summarizing these dynamics from the view point of banking industry, one observes that the profitability of non-fixed rate mortgages suffers from a high interest level as well as from a low interest level.

The challenge of funding non-fixed rate mortgages is seen in optimizing the monthly funding activities taking into account the stochasticity of interest rate dynamics and mortgage volume. Depending on the asset and liability structure of a bank, the non-fixed rate mortgages are funded to a certain extent with bonds of different maturities. The range of these maturities may vary between one month and 10 years. Taking into account the liquidity with respect to which the various maturities are traded, it is observed that short term bonds, say bonds with a maturity of up to 1 year, may be borrowed in a considerably larger volume than bonds with a maturity beyond 2 years. In addition, the short term rates are much more volatile than long term rates.

The funding problem, referred to below, has been introduced in Frauendorfer 1996 [24] and is discussed with respect to interest rate models in Frauendorfer 
and Schürle 1997 [26]. Herein, this problem is investigated with respect to its solvability.

Let $\mathcal{D}:=\{1,2, \ldots, D\}$ be the set of prescribed times at which bonds mature. Taking into account the liquidity within the various maturities, only a subset $\mathcal{D}^{S} \subset \mathcal{D}$ is regarded for funding. Furthermore, it is assumed that the bonds are held until maturity, so that changes in the price of a bond during the holding period may be relaxed. The volume of bonds borrowed at time $t=0, \ldots, T$ with maturity $d \in \mathcal{D}$ is denoted $v_{t}^{d,+} ; v_{t}^{d}$ represents the total volume of bonds with maturity $d$ at time $t$. Clearly, $v_{t}^{d}$ is determined by

$$
\begin{array}{ll}
v_{t}^{d}=v_{t-1}^{d+1}+v_{t}^{d,+} & t=0,1, \ldots, T ; \forall d \in \mathcal{D}^{S} \\
v_{t}^{d}=v_{t-1}^{d+1} & t=0,1, \ldots, T ; \forall d \notin \mathcal{D}^{S} .
\end{array}
$$

The total funding volume at $t$ is given with

$$
x_{t}=\sum_{d \in \mathcal{D}} v_{t}^{d} \quad t=0, \ldots, T .
$$

Its evolvement over time is determined with the stochastic change $\xi_{t} \in \mathbb{R}$,

$$
x_{t}=x_{t-1}+\xi_{t} \quad t=1,2, \ldots, T .
$$

The term structure dynamics may be represented by a finite-dimensional discretetime stochastic process $\eta_{t} \in \mathbb{R}^{K} ; t=0, \ldots, T$. Let the accrued interest payments of an unit borrowed with maturity $d$ be denoted $\rho_{t}\left(\eta_{t}, d\right)$. Setting $v_{t}:=\left(v_{t}^{d} ; d \in\right.$ $\mathcal{D}), v_{t}^{+}:=\left(v_{t}^{d,+} ; d \in \mathcal{D}^{S}\right)$ allows for writing the accrued interest paymenets as inner product within $\mathbb{R}^{\left|\mathcal{D}^{S}\right|}$ :

$$
<\rho_{t}\left(\eta_{t}\right), v_{t}^{+}>=\sum_{d \in \mathcal{D}^{S}} \rho_{t}\left(\eta_{t}, d\right) \cdot v_{t}^{d,+}
$$

The stochastic multistage linear program which minimizes the expected present value of interest payments reads as

$$
\begin{array}{lll}
\min \int_{\Omega} \sum_{t=0}^{T}<\rho_{t}\left(\eta_{t}\right), v_{t}^{+}>d P(\eta, \xi) & & \\
v_{t}^{d}-v_{t-1}^{d+1}-v_{t}^{d,+} & =0 & \forall t, \forall d \in \mathcal{D}^{S} \\
v_{t}^{d}-v_{t-1}^{d+1} & =0 & \forall t, \forall d \notin \mathcal{D}^{S} \\
x_{t}-\sum_{d \in \mathcal{D}} v_{t}^{d} & =0 & \forall t \\
x_{t}-x_{t-1} & =\xi_{t} & \forall t \\
v_{t}^{d,+} \geq 0 ; v_{t}^{d}, x_{t} \in \mathbb{R} \text { nonanticipative } & & \forall t, \forall d \in \mathcal{D} .
\end{array}
$$

Observe that the left-hand side of the constraints (5) is deterministic. This fact requires that the stochastic interest payments, that clearly depend on the funding decision, has to be incorporated in the dynamis of $\xi$. To preserve convexity 
one has to relax the stochastic dependency of the volume change on the decisions. Then probability measures must be unaffected by the decisions taken. The stochastic interest payments appear in the objective, only. If, in addition, the conditional probabilities $P_{t}\left(\cdot \mid \eta_{t-1}, \xi_{t-1}\right)$ depend linearly on the observation in period $t-1$, the convexity of the stochastic multistage program (5) is preserved and the value function of stage $t$ associated with (5) are saddle functions concave-convex in $\left(\eta_{t}, \xi_{t}\right)$ (see Frauendorfer 1994/1996 [22], [23]).

The underlying saddle structure of the value functions motivates the application of the barycentric approximation (see Figure (1) and section 3), which optimizes the discretization of the stochastic interest rate and volume processes.
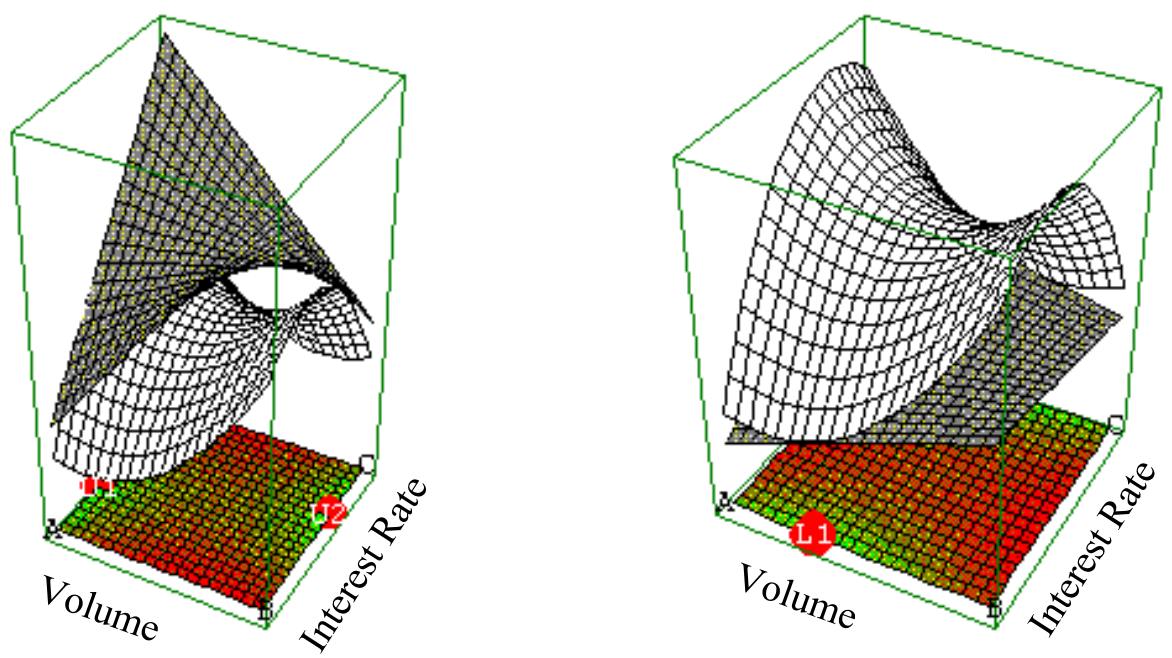

Figure 1: Bilinear approximation of the value function

An illustration of a scenario tree is given in Figure 2.

It is further noted that +1 and -1 are the only non-zero coefficients of the matrix on the left-hand side of $(5)$. The submatrices associated with the various stages $t=1, \ldots, T$ remain unchanged and are of high sparsity. In addition to the low dimensions of the term structure representation, the dynamics of the stochastic right-hand side is characterized by a one-dimensional discrete-time process. All these properties increase the solvability of the underlying problem considerably, as these are exploited by sophisticated algorithms.

Due to the achieved progress in the methodological developments within mathematical programming stochastic programming has received increasing attention in finance. At this place it is referred to the successful and valuable contributions of Dantzig 1990/1993 [13], [14], Ziemba 1975/1986/1992/1994/1997 [57], [36], [55], [9], [56], [42], Dempster 1997 [11], Klaassen 1997 [35], Dupačová 1991/1992/1997 [15], [16] [1], Mulvey 1992/1994/1996/1997 [41], [37], [39], [56], 


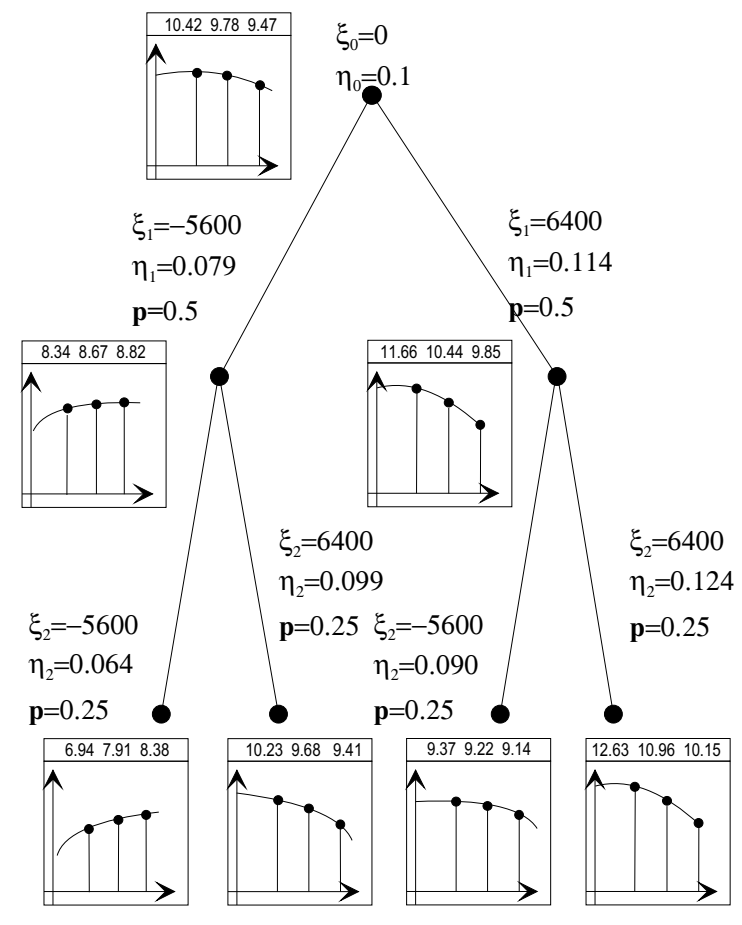

Figure 2: Scenario tree for 2-stage funding problem with interest rate curve being driven by three maturities.

[42], Zenios 1991/1992/1993/1995/1996 [52], [53],[54], [55], [12] [28], [50], [43], Hiller and Eckstein 1994 [29], and Cariño et al. 1994/1995/1997 [9], [10], [8].

\section{Barycentric scenario trees}

In the dynamic formulation (2),

$$
\int \phi_{t}\left(u_{t-1}, \eta_{t}, \xi_{t}\right) d P_{t}\left(\eta_{t}, \xi_{t} \mid \omega_{t-1}\right)
$$

has to be evaluated within the nested optimization, which encounters a serious challenge. As said, in the convex case the value functions are saddle functions in (1). This motivates the application of the barycentric approximation technique, which not only helps carry out the nested optimization, but also provides information on how accurate the real dynamics are mapped.

In this section, the barycentric approximation technique is shortly reviewed, illustrating how the multidimensional integration is bounded from above and below. For details it is referred to Frauendorfer 1992/1996 [21], [23].

Since supports are compact, the stochastic outcomes $\omega_{t}:=\left(\eta_{t}, \xi_{t}\right)$ may be covered 
by a $\times$-simplex ${ }^{1} \Omega_{t}:=\Theta_{t} \times \Xi_{t}$ for $t=1, \ldots, T$. Taking into account the dependency of $\left(\eta_{t}, \xi_{t}\right)$ on $\left(\eta_{t-1}, \xi_{t-1}\right)$, one may select the $\times$-simplicial coverage conditioned on $\omega_{t-1}=\left(\eta_{t-1}, \xi_{t-1}\right), \Omega_{t}\left(\omega_{t-1}\right):=\Theta_{t}\left(\omega_{t-1}\right) \times \Xi_{t}\left(\omega_{t-1}\right)$.

To a given scenario tree $A$ of the form (4) and its projections $A^{t}$ for $t=1, \ldots, T$, a $\times$-simplicial partition of the support of $\omega_{t}$ may be selected for any $\omega_{t-1} \in$ $A_{t-1}\left(\omega_{t-2}\right)$ :

$$
\begin{aligned}
C_{t}\left(\omega_{t-1}\right):= & \left\{\Omega_{t, i_{t}}\left(\omega_{t-1}\right) \mid \cup_{i_{t}=1}^{I_{t}} \Omega_{t, i_{t}}\left(\omega_{t-1}\right)=\Omega_{t}\left(\omega_{t-1}\right) \supset \operatorname{supp} P_{t}\left(\cdot \mid \omega_{t-1}\right),\right. \\
& \left.\Omega_{t, i_{t}^{\prime}}\left(\omega_{t-1}\right) \cap \Omega_{t, i_{t}^{\prime \prime}}\left(\omega_{t-1}\right)=\emptyset, \forall i_{t}^{\prime} \neq i_{t}^{\prime \prime}, i_{t}=1, \ldots, I_{t}\right\} .
\end{aligned}
$$

We call the resulting set process $C:=\left(C_{t}\left(\omega_{t-1}\right) ; t=1, \ldots, T\right)$ a $\times$-simplicial coverage process consistent with $A$ iff $A_{t}\left(\omega_{t-1}\right) \subset \Omega_{t}\left(\omega_{t-1}\right)$ for all $\omega_{t-1} \in A_{t-1}\left(\omega_{t-2}\right)$, $t=1, \ldots, T$.

For the ease of exposition, we first consider a partition $C_{t}\left(\omega_{t-1}\right)$ consists of one $\times$-simplex, i.e.: $C_{t}\left(\omega_{t-1}\right):=\Omega_{t}\left(\omega_{t-1}\right):=\Theta_{t}\left(\omega_{t-1}\right) \times \Xi_{t}\left(\omega_{t-1}\right) . \Theta_{t}\left(\omega_{t-1}\right), \Xi_{t}\left(\omega_{t-1}\right)$ are regular simplices in $\mathbb{R}^{K}, \mathbb{R}^{L}$ with associated vertices $a_{t, \nu}$ and $b_{t, \mu}, \nu=$ $0, \ldots, K, \mu=0, \ldots, L$. Below, $\lambda_{\mu}\left(\eta_{t}\right)$ and $\tau_{\nu}\left(\xi_{t}\right)$ denote the barycentric weights of $\eta_{t}$ and $\xi_{t}$ with respect to the simplices $\Theta_{t}\left(\omega_{t-1}\right)$ and $\Xi_{t}\left(\omega_{t-1}\right)$.

The conditional probability measure $P_{t}\left(\cdot \mid \omega_{t-1}\right)$ induces mass distribution $\mathcal{M}_{t, \nu}$ with corresponding generalized barycenter

$$
\xi_{t, \nu}:=\sum_{\mu} b_{t, \mu} \frac{\int \lambda_{\nu}\left(\eta_{t}\right) \cdot \tau_{\mu}\left(\xi_{t}\right) d P_{t}\left(\eta_{t}, \xi_{t} \mid \omega_{t-1}\right)}{\int \lambda_{\nu}\left(\eta_{t}\right) d P_{t}\left(\eta_{t}, \xi_{t} \mid \omega_{t-1}\right)}
$$

on the (L-dimensional) simplices $\left\{a_{t, \nu}\right\} \times \Xi_{t}\left(\omega_{t-1}\right){ }^{2}$ For $\nu=0, \ldots, K$ let the mass

$$
\mathcal{M}_{t, \nu}\left(\left\{a_{\nu}\right\} \times \Xi_{t}\left(\omega_{t-1}\right)\right):=\int \lambda_{\nu}\left(\eta_{t}\right) d P_{t}\left(\eta_{t}, \xi_{t} \mid \omega_{t-1}\right)
$$

be assigned to $\left(a_{t, \nu}, \xi_{t, \nu}\right)$. As for $\nu=0, \ldots, K$ the mass distributions $\mathcal{M}_{t, \nu}$ add up to a conditional probability distribution, one gets a discrete conditional probability measure $Q_{t}^{l}\left(\cdot \mid \omega_{t-1}\right)$ on $\Theta_{t}\left(\omega_{t-1}\right) \times \Xi_{t}\left(\omega_{t-1}\right)$.

Due to symmetry, the conditional probability measure $P_{t}\left(\cdot \mid \omega_{t-1}\right)$ induces mass distributions $\mathcal{M}_{t, \mu}$ with associated generalized barycenters

$$
\eta_{t, \mu}:=\sum_{\nu} a_{t, \nu} \frac{\int \lambda_{\nu}\left(\eta_{t}\right) \cdot \tau_{\mu}\left(\xi_{t}\right) d P_{t}\left(\eta_{t}, \xi_{t} \mid \omega_{t-1}\right)}{\int \tau_{\mu}\left(\xi_{t}\right) d P_{t}\left(\eta_{t}, \xi_{t} \mid \omega_{t-1}\right)}
$$

on the $(K$-dimensional $)$ simplices $\Theta_{t}\left(\omega_{t-1}\right) \times\left\{b_{t, \mu}\right\}$. Again, for $\mu=0, \ldots, L$ let the mass

$$
\mathcal{M}_{t, \mu}\left(\Theta_{t}\left(\omega_{t-1}\right) \times\left\{b_{t, \mu}\right\}\right):=\int \tau_{\mu}\left(\xi_{t}\right) d P_{t}\left(\eta_{t}, \xi_{t} \mid \omega_{t-1}\right)
$$

\footnotetext{
${ }^{1} \mathrm{~A} \times$-simplex is a set whose closure is representable as a Cartesian product of simplices.

${ }^{2}$ In the notation it is omitted for simplicity that the vertices and generalized barycenters depend on $\omega_{t-1}$.
} 
be assigned to the points $\left(\eta_{t, \mu}, b_{t, \mu}\right)$. Analogously, for $\mu=0, \ldots, L$ the mass distributions $\mathcal{M}_{t, \mu}$ add up to a conditional probability distribution, yielding a discrete conditional probability measure $Q_{t}^{u}\left(\cdot \mid \omega_{t-1}\right)$ on $\Theta_{t}\left(\omega_{t-1}\right) \times \Xi_{t}\left(\omega_{t-1}\right)$.

As it becomes obvious from (8),(9),(10) and (11), the advantagous feature from a computational viewpoint is that generalized barycenters and their probabilities are completely determined by the first moments of $\eta_{t}$ and $\xi_{t}$, and by the cross moments $\mathrm{E}\left(\eta_{t} \cdot \xi_{t}\right)$.

It has been proven in Frauendorfer 1992 [21] that in the convex case the expression in (6) is approximated from below and above by

$$
\begin{aligned}
& \left(\mathrm{E}_{t}^{l} \phi_{t}\right)\left(u_{t-1}, \omega_{t-1}\right) \quad:=\int \phi_{t}\left(u_{t-1}, \omega_{t}\right) d Q_{t}^{l}\left(\omega_{t} \mid \omega_{t-1}\right) \leq \\
& \leq\left(\mathrm{E}_{t} \phi_{t}\right)\left(u_{t-1}, \omega_{t-1}\right):=\int \phi_{t}\left(u_{t-1}, \omega_{t}\right) d P_{t}\left(\omega_{t} \mid \omega_{t-1}\right) \leq \\
& \leq\left(\mathrm{E}_{t}^{u} \phi_{t}\right)\left(u_{t-1}, \omega_{t-1}\right):=\int \phi_{t}\left(u_{t-1}, \omega_{t}\right) d Q_{t}^{u}\left(\omega_{t} \mid \omega_{t-1}\right)
\end{aligned}
$$

This technique has been termed barycentric approximation and can easily be applied to a partition of the form (7). For details it is referred to Frauendorfer 1992/1994/1996 [21], [22] and [23].

Let be defined

$$
\begin{aligned}
& A_{t}^{l}\left(\omega_{t-1}\right):=\operatorname{supp} Q_{t}^{l}\left(\cdot \mid \omega_{t-1}\right), \\
& A_{t}^{u}\left(\omega_{t-1}\right):=\operatorname{supp} Q_{t}^{u}\left(\cdot \mid \omega_{t-1}\right) .
\end{aligned}
$$

Clearly, $A_{0}^{l}=A_{0}^{u}=\omega_{0}$. Starting with a partition $C_{1}^{l}\left(\omega_{0}\right)=C_{1}^{u}\left(\omega_{0}\right)$, the associated barycentric approximation yields $Q_{1}^{l}\left(\omega_{0}\right)$ and $Q_{1}^{u}\left(\omega_{0}\right)$ and the associated supports $A_{1}^{l}\left(\omega_{0}\right)$ and $A_{1}^{u}\left(\omega_{0}\right)$. Hence, the partitions $C_{t}^{l}\left(\omega_{t-1}\right)$ and $C_{t}^{u}\left(\omega_{t-1}\right)$ are selected with respect to the support of $Q_{t}^{l}\left(\cdot \mid \omega_{t-1}\right)$ and $Q_{t}^{u}\left(\cdot \mid \omega_{t-1}\right)$ in an inductive manner. This way, the barycentric scenario trees and the associated path probabilities are given in analogy to (4):

$$
\begin{array}{r}
A^{l}:=\left\{\beta^{l} \mid \beta_{t}^{l} \in A_{t}^{l}\left(\beta_{t-1}^{l}\right), \forall t \geq 1\right\}, \\
Q^{l}\left(\beta^{l}\right):=\prod_{t=1}^{T} Q_{t}^{l}\left(\beta_{t}^{l} \mid \beta_{t-1}^{l}\right),
\end{array}
$$

and

$$
\begin{array}{r}
A^{u}:=\left\{\beta^{u} \mid \beta_{t}^{u} \in A_{t}^{u}\left(\beta_{t-1}^{u}\right), \forall t \geq 1\right\}, \\
Q^{u}\left(\beta^{u}\right):=\prod_{t=1}^{T} Q_{t}^{u}\left(\beta_{t}^{u} \mid \beta_{t-1}^{u}\right) .
\end{array}
$$

$A^{t, l}, A^{t, u}$ denotes the projection of $A^{l}, A^{u}$ onto $[0, t]$, and $\beta^{l}=\left(\beta_{0}^{l}, \ldots, \beta_{T}^{l}\right), \beta^{u}=$ $\left(\beta_{0}^{u}, \ldots, \beta_{T}^{u}\right)$ the associated barycentric scenarios. Note that due to the construction the barycentric scenario trees, $A^{l}, A^{u}$ and the associated $\times$-simplicial coverage processes $C^{l}, C^{u}$ are consistent. 
Substituting in (1) the probability measure $P$ by its discretizations $Q^{l}$ and $Q^{u}$ yields the associated stochastic multistage programs

$$
\begin{array}{ll}
\min & \int\left[\sum_{t=0}^{T} \rho_{t}\left(u_{t}, \eta_{t}\right)\right] d Q^{l}(\eta, \xi) \\
\text { s.t. } & f_{t}\left(u_{t-1}, u_{t}\right) \leq h_{t}\left(\xi_{t}\right), \quad t=0,1, \ldots, T,
\end{array}
$$

and

$$
\begin{array}{ll}
\min & \int\left[\sum_{t=0}^{T} \rho_{t}\left(u_{t}, \eta_{t}\right)\right] d Q^{u}(\eta, \xi) \\
\text { s.t. } & f_{t}\left(u_{t-1}, u_{t}\right) \leq h_{t}\left(\xi_{t}\right), \quad t=0,1, \ldots, T .
\end{array}
$$

The associated value functions are given through the dynamic formulation

$$
\begin{aligned}
& \psi_{t}\left(u_{t-1}, \eta_{t}, \xi_{t}\right):=\min \rho_{t}\left(u_{t}, \eta_{t}\right)+\int \psi_{t+1}\left(u_{t}, \omega_{t+1}\right) d Q_{t}^{l}\left(\omega_{t+1} \mid \eta_{t}, \xi_{t}\right) \\
& \text { s.t. } \quad f_{t}\left(u_{t-1}, u_{t}\right) \leq h_{t}\left(\xi_{t}\right),
\end{aligned}
$$

and

$$
\begin{aligned}
& \Psi_{t}\left(u_{t-1}, \eta_{t}, \xi_{t}\right):=\min \rho_{t}\left(u_{t}, \eta_{t}\right)+\int \Psi_{t+1}\left(u_{t}, \omega_{t+1}\right) d Q_{t}^{u}\left(\omega_{t+1} \mid \eta_{t}, \xi_{t}\right) \\
& \text { s.t. } \quad f_{t}\left(u_{t-1}, u_{t}\right) \leq h_{t}\left(\xi_{t}\right),
\end{aligned}
$$

with $\psi_{T+1}(\cdot)=\Psi_{T+1}(\cdot):=0$.

It has been proven in Frauendorfer 1994 [22] that (20) and (21) provide lower and upper approximates for the value function $\phi_{t}\left(u_{t-1}, \eta_{t}, \xi_{t}\right)$ with $\psi_{t}\left(u_{t-1}, \eta_{t}, \xi_{t}\right)$ and $\Psi_{t}\left(u_{t-1}, \eta_{t}, \xi_{t}\right)$, i.e:

$$
\psi_{t}\left(u_{t-1}, \eta_{t}, \xi_{t}\right) \leq \phi_{t}\left(u_{t-1}, \eta_{t}, \xi_{t}\right) \leq \Psi_{t}\left(u_{t-1}, \eta_{t}, \xi_{t}\right)
$$

Solving (18) and (19) yields policies $u^{l}:=\left(u_{0}^{l}, u_{1}^{l}, \ldots, u_{T}^{l}\right)$ and $u^{u}:=\left(u_{0}^{u}, u_{1}^{u}, \ldots, u_{T}^{u}\right)$ where $u_{t}^{l}, u_{t}^{u}$ denote the decisions made after $\beta^{t, l} \in A^{t, l}, \beta^{t, u} \in A^{t, u}, \quad(t=$ $1, \ldots, T)$ is observed. Hence, upper and lower approximations of the value functions may correspond to different policies. Both $\psi_{t}(\cdot)$ and $\Psi_{t}(\cdot)$ epiconverge to $\phi_{t}(\cdot)$ in case the weak convergence of the conditional discrete probability measures $Q_{t}^{l}\left(\cdot \mid \beta_{t-1}^{l}\right), Q_{t}^{u}\left(\cdot \mid \beta_{t-1}^{u}\right)$ to $P_{t}\left(\cdot \mid \beta_{t-1}^{l}\right), P_{t}\left(\cdot \mid \beta_{t-1}^{u}\right)$, respectively, is ensured for $t=1, \ldots, T$. This requires that the sub- $\times$-simplices of the coverage processes become arbitrarily small with respect to their diameters. In the following section we discuss in what way this convergence can be monitored, taking into account that lower and upper approximation refer to different policies and different coverage processes. 


\section{Error analysis}

As mentioned in the introduction, the difficulty in solving stochastic linear multistage programs is seen in the nested optimization and multidimensional integration of implicitely given value functions. Discretizing the conditional probability measures in the barycentric sense helps to overcome these difficulties and, in the convex case, provides approximate policies for the underlying problem including bounds on the optimal expected value. However, lower and upper approximation of the value functions refer to different scenario trees and associated consistent $\times$-simplicial coverage processes, which only coincide with respect to the data at $t=0$. For assessing how the inaccuracy of the approximation evolves over time, based on which the refinement process can be monitored efficiently, additional information has to be determined.

For the ease of exposition, the following notations are used:

$$
\begin{aligned}
\left(\mathrm{E}_{t}^{l} \Psi_{t}\right)\left(u_{t-1}, \omega_{t-1}\right) & :=\int \Psi_{t}\left(u_{t-1}, \omega_{t}\right) d Q_{t}^{l}\left(\omega_{t} \mid \omega_{t-1}\right) \\
\left(\mathrm{E}_{t}^{u} \Psi_{t}\right)\left(u_{t-1}, \omega_{t-1}\right) & :=\int \Psi_{t}\left(u_{t-1}, \omega_{t}\right) d Q_{t}^{u}\left(\omega_{t} \mid \omega_{t-1}\right) \\
\left(\mathrm{E}_{t}^{l} \psi_{t}\right)\left(u_{t-1}, \omega_{t-1}\right) & :=\int \psi_{t}\left(u_{t-1}, \omega_{t}\right) d Q_{t}^{l}\left(\omega_{t} \mid \omega_{t-1}\right) \\
\left(\mathrm{E}_{t}^{u} \psi_{t}\right)\left(u_{t-1}, \omega_{t-1}\right) & :=\int \psi_{t}\left(u_{t-1}, \omega_{t}\right) d Q_{t}^{u}\left(\omega_{t} \mid \omega_{t-1}\right) \\
\beta_{\mathrm{t}+1}^{\mathrm{l}} \in \mathrm{A}_{\mathrm{t}+1}^{\mathrm{l}}\left(\beta_{\mathrm{t}}^{\mathrm{l}}\right) & \cdot \boldsymbol{u}_{\mathrm{t}}^{\mathrm{l}} \cdot \psi_{\mathrm{t}-1}^{\mathrm{l}}\left(\mathrm{u}_{\mathrm{t}-1}^{\mathrm{l}}, \beta_{\mathrm{t}}^{\mathrm{l}}\right)
\end{aligned}
$$

Figure 3: Scenario tree corresponding to the lower approximation

Let the scenario trees $A^{l}, A^{u}$ and the associated consistent coverage processes $C^{l}, C^{u}$ be and the associated stochastic multistage programs (18) and (19) be solved. Given a scenario $\beta^{t, l}=\left(\beta_{1}^{l}, \ldots, \beta_{t}^{l}\right) \in A^{t, l}$ up to $t$, decision $u_{t}^{l}$ and its value $\psi_{t}\left(u_{t-1}^{l}, \beta_{t}^{l}\right)$ is available. The stochastic evolvement during the next period is approximated by the conditional barycentric discretization $\beta_{t+1}^{l} \in A_{t+1}^{l}\left(\beta_{t}^{l}\right)$ with the associated minimal value $\psi_{t+1}\left(u_{t}^{l}, \beta_{t+1}^{l}\right)$ (see Figure 3 ); analogoulsy, for $\beta^{t, u} \in A^{t, u}$ the decision $u_{t}^{u}$ and the value $\Psi_{t}\left(u_{t-1}^{u}, \beta_{u}^{t}\right)$ is known. The stochastic evolvement during the next period is approximated by the conditional barycentric 


$$
\beta_{t+1}^{u} \in A_{t+1}^{u}\left(\beta_{t}^{u}\right) ! \Psi_{t}^{u}\left(u_{t-1}^{u}, \beta_{t}^{u}\right)
$$

Figure 4: Scenario tree corresponding to the upper approximation

discretization $\beta_{t+1}^{u} \in A_{t+1}^{u}\left(\beta_{t}^{u}\right)$ with the associated minimal values $\Psi_{t+1}\left(u_{t}^{u}, \beta_{t+1}^{u}\right)$ (see Figure 4). Due to (22), the following inequalities hold :

$$
\begin{aligned}
\psi_{t}\left(u_{t-1}^{l}, \beta_{t}^{l}\right) & \leq \phi_{t}\left(u_{t-1}^{l}, \beta_{t}^{l}\right) \\
\phi_{t}\left(u_{t-1}^{u}, \beta_{t}^{u}\right) & \leq \Psi_{t}\left(u_{t-1}^{u}, \beta_{t}^{u}\right) .
\end{aligned}
$$

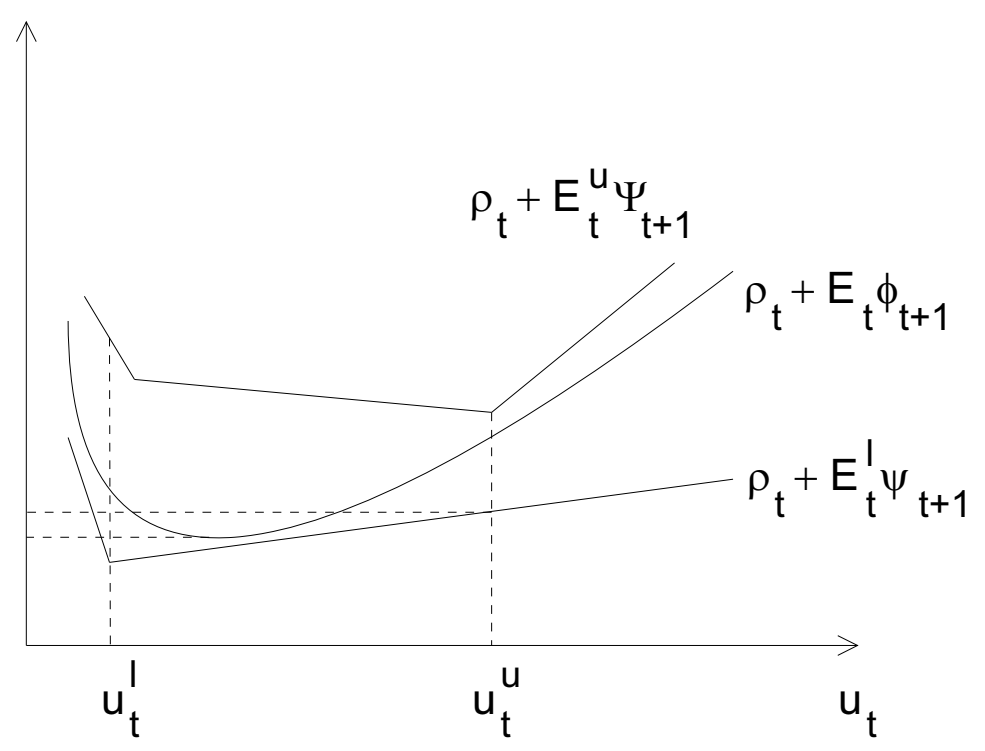

Figure 5: Lower and upper approximation for $\rho_{t}+\mathrm{E}_{t} \phi_{t+1}$

The missing upper bound for $\phi_{t}\left(u_{t-1}^{l}, \beta_{t}^{l}\right)$ is available with the evaluation of $\Psi_{t}\left(u_{t-1}^{l}, \beta_{t}^{l}\right)$, the missing lower bound for $\phi_{t}\left(u_{t-1}^{u}, \beta_{t}^{u}\right)$ can be obtained with $\Psi_{t}\left(u_{t-1}^{u}\right.$, $\left.\beta_{t}^{u}\right)$. In both cases, a $(T-t)$-stage multistage program must be solved with respect to the barycentric scenario trees conditioned on $\beta_{t}^{l}, \beta_{t}^{u}$, respectively, providing the inner approximation, the outer approximation, respectively. Due to the convexity of the value function $\phi_{t}$ in the decision $u_{t}$, we focus on the minimizer of the outer approximation: This becomes evident from Figure 5: Evaluating the 
inner approximation at the minimizer $u_{t}^{l}$ of the outer approximation provides a further bound on $\phi_{t}\left(u_{t-1}^{l}, \beta_{t}^{l}\right)$, i.e.:

$$
\beta_{t+1}^{l} \in A_{t+1}^{l}\left(\beta_{t}^{l}\right) u_{t}^{\prime}\left(u_{t-1}^{l}, \beta_{t}^{l}\right)
$$

Figure 6: Evaluation of the upper approximation $\Psi_{t}\left(u_{t-1}^{l}, \beta_{t}^{l}\right)$ corresponding to each node of the scenario tree $A^{l}$.

$$
\phi_{t}\left(u_{t-1}^{l}, \beta_{t}^{l}\right) \leq\left[\rho_{t}+\mathrm{E}_{t}^{u} \Psi_{t+1}\right]\left(u_{t}^{l}, \beta_{t}^{l}\right)
$$

Note that $u_{t}^{l}$ solves (20) and is feasible for (21) given $u_{t-1}^{l}$ and $\beta_{t}^{l}$ (see Figure 6).

To the contrary, the value of the outer approximation at the minimizer $u_{t}^{u}$ of the inner approximation does not necessarily bound $\phi_{t}(\cdot)$ from below (see Figure 5 ), i.e.:

$$
\left[\rho_{t}+\mathrm{E}_{t}^{l} \psi_{t+1}\right]\left(u_{t}^{u}, \beta_{t}^{u}\right) \not \underline{ } \phi_{t}\left(u_{t-1}^{u}, \beta_{t}^{u}\right)=\min \left[\rho_{t}+E_{t} \phi_{t+1}\right]\left(u_{t}, \beta_{t}^{u}\right)
$$

Note that $u_{t}^{u}$ solves (21) and is feasible for (20) given $u_{t-1}^{u}$ and $\beta_{t}^{u}$.

$$
\beta_{t+1}^{l} \in A_{t+1}^{l}\left(\beta_{t}^{l}\right) \psi_{t}\left(u_{t-1}^{l}, \beta_{t}^{l}\right), \Psi_{t}\left(u_{t-1}^{l}, \beta{ }_{t}^{l}\right)
$$

Figure 7: Information known in period $t$

Above observations let us focus on the barycentric scenario tree which corresponds to the lower approximation (see Figure 7), for which immediately error bounds can be obtained at any node of that tree by

$$
\epsilon_{t}\left(\beta_{t}^{l}\right):=\Psi_{t}\left(u_{t-1}^{l}, \beta_{t}^{l}\right)-\psi_{t}\left(u_{t-1}^{l}, \beta_{t}^{l}\right)
$$




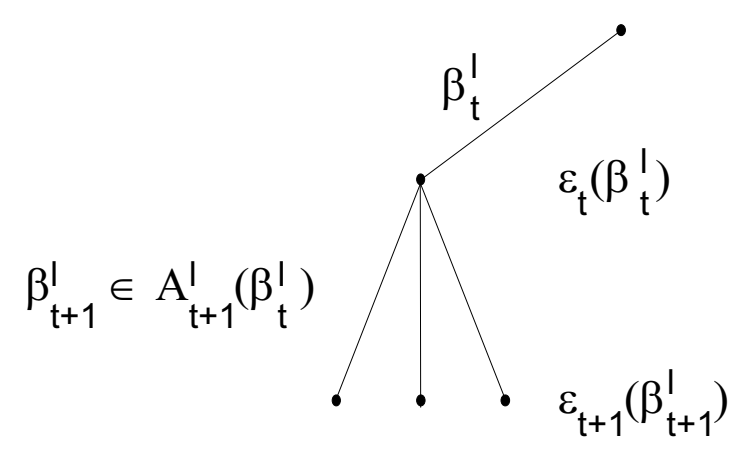

Figure 8: Error bounds at the nodes of $\beta_{t}^{l}$

The evaluation of the error at each period provides a measure for the goodness of the lower approximation (see Figure 8). If the error at some node in period $t$ is zero, the approximation of the value function $\phi_{t}(\cdot)$ and their minimizers is exact and does not need to be improved beyond this node. Clearly, in case that $\epsilon_{t}\left(\beta_{t}^{l}\right)$ is positive at some node, than the error bounds prior to that node are positive, too.

By definition $\epsilon_{T+1} \equiv 0$. Moving backwards in time, the error bounds increase. The integration error occurs first and, hence, causes increasing error within the nested minimization and integration procedure. We shall investigate here how the error bound $\epsilon_{t}(\cdot)$ evolves. For this purpose, we summarize the inequalities (24) and (26) according to

$$
\begin{aligned}
{\left[\rho_{t}+\mathrm{E}_{t}^{l} \psi_{t+1}\right]\left(u_{t}^{l}, \beta_{t}^{l}\right) } & =\psi_{t}\left(u_{t-1}^{l}, \beta_{t}^{l}\right) \leq \phi_{t}\left(u_{t-1}^{l}, \beta_{t}^{l}\right) \leq \\
& \leq \Psi_{t}\left(u_{t-1}^{l}, \beta_{t}^{l}\right)=\left[\rho_{t}+\mathrm{E}_{t}^{u} \Psi_{t+1}\right]\left(\hat{u}_{t}^{u}, \beta_{t}^{l}\right) \leq \\
& \leq\left[\rho_{t}+\mathrm{E}_{t}^{u} \Psi_{t+1}\right]\left(u_{t}^{l}, \beta_{t}^{l}\right)
\end{aligned}
$$

where $\hat{u}_{t}^{u}$ solves (21) given $u_{t-1}^{l}$ and $\beta_{t}^{l}$. Obviously,

$$
\left[\rho_{t}+\mathrm{E}_{t}^{l} \psi_{t+1}\right]\left(u_{t}^{l}, \beta_{t}^{l}\right) \leq\left[\rho_{t}+\mathrm{E}_{t}^{u} \Psi_{t+1}\right]\left(u_{t}^{l}, \beta_{t}^{l}\right),
$$

however

$$
\left[\rho_{t}+\mathrm{E}_{t}^{l} \Psi_{t+1}\right]\left(u_{t}^{l}, \beta_{t}^{l}\right) \not \subset\left[\rho_{t}+\mathrm{E}_{t}^{u} \Psi_{t+1}\right]\left(u_{t}^{l}, \beta_{t}^{l}\right),
$$

as the approximate value function $\Psi_{t+1}(\cdot)$ need not necessarily be a saddle function. For the evaluation of $\left[\mathrm{E}_{t}^{l} \Psi_{t+1}\right]\left(u_{t}^{l}, \beta_{t}^{l}\right)$ and $\left[\mathrm{E}_{t}^{u} \Psi_{t+1}\right]\left(u_{t}^{l}, \beta_{t}^{l}\right)$ it is referred to Figures 9 and 10. Therefore,

$$
\delta_{t}\left(\beta_{t}^{l}\right):=\left[\mathrm{E}_{t}^{u} \Psi_{t+1}\right]\left(u_{t}^{l}, \beta_{t}^{l}\right)-\left[\mathrm{E}_{t}^{l} \Psi_{t+1}\right]\left(u_{t}^{l}, \beta_{t}^{l}\right),
$$

may also become negative. Accepting $\delta_{t}\left(\beta_{t}^{l}\right)$ as error estimate associated with the integration of $\Psi_{t+1}(\cdot)$ (see Figure 11), we conclude that in case $\delta_{t}\left(\beta_{t}^{l}\right) \gg 0$, the 


$$
\begin{aligned}
& \text {. } \mathrm{u}_{\mathrm{t}-1}^{\mathrm{l}} \\
& \beta_{\mathrm{t}}^{\mathrm{l}} \\
& u_{t}^{\prime}, \quad\left[E_{t}^{u} \Psi_{t+1}\right]\left(u_{t}^{\prime}, \beta_{t}^{\prime}\right) \\
& \beta_{\mathrm{t}+1}^{\mathrm{u}} \in \mathrm{A}_{\mathrm{t}+1}^{\mathrm{u}}\left(\beta_{\mathrm{t}}^{\mathrm{l}}\right) \\
& \text { - . } \Psi_{t+1}\left(u_{t}^{l}, \beta_{t+1}^{u}\right)
\end{aligned}
$$

Figure 9: Evaluation of $\left[\mathrm{E}_{t}^{u} \Psi_{t+1}\right]\left(u_{t}^{l}, \beta_{t}^{l}\right)$

$$
\beta_{t+1}^{l} \in A_{t+1}^{l}\left(\beta_{t}^{l}\right) u_{t+1}^{\prime}\left(u_{t}^{l}, \beta_{t+1}^{l}\right)
$$

Figure 10: Evaluation of $\left[\mathrm{E}_{t}^{l} \Phi_{t+1}\right]\left(u_{t}^{l}, \beta_{t}^{l}\right)$

$$
\beta_{t+1}^{I} \in A_{t+1}^{I}\left(\beta_{t}^{I}\right)
$$

Figure 11: Error increments at the nodes of $A^{l}$

current discretization has caused an inaccurate integration of $\Psi_{t+1}(\cdot)$ and, hence, of $\phi_{t+1}(\cdot)$. In case that $\delta_{t}\left(\beta_{t}^{l}\right)$ is even negative, the current approximation of the saddle function $\phi_{t+1}(\cdot)$ by $\Psi_{t+1}(\cdot)$ cannot be accepted as sufficiently accurate, as $\Psi_{t+1}(\cdot)$ does not even satisfy the saddle property. 


\begin{tabular}{|l|r|r|r|}
\hline \# ref. & upper bound & lower bound & accuracy \\
\hline 0 & 6694.96 & 5906.92 & $11.77 \%$ \\
\hline 1 & 6694.96 & 6268.61 & $6.37 \%$ \\
\hline 2 & 6694.96 & 6269.41 & $6.36 \%$ \\
\hline 3 & 6694.96 & 6305.73 & $5.81 \%$ \\
\hline
\end{tabular}

Table 1: Lower and upper bounds for the first 3 refinements

We derive in what way $\delta_{t}\left(\beta_{t}^{l}\right)$ contributes to $\epsilon_{t}\left(\beta_{t}^{l}\right)$. This comes immediate from

$$
\begin{aligned}
\epsilon_{t}\left(\beta_{t}^{l}\right) & =\Psi_{t}\left(u_{t-1}^{l}, \beta_{t}^{l}\right)-\psi_{t}\left(u_{t-1}^{l}, \beta_{t}^{l}\right) \leq \\
& \leq\left[\rho_{t}+\mathrm{E}_{t}^{u} \Psi_{t+1}\right]\left(u_{t}^{l}, \beta_{t}^{l}\right)-\left[\rho_{t}+\mathrm{E}_{t}^{l} \psi_{t+1}\right]\left(u_{t}^{l}, \beta_{t}^{l}\right)= \\
& =\mathrm{E}_{t}^{u} \Psi_{t+1}\left(u_{t}^{l}, \beta_{t}^{l}\right)-\mathrm{E}_{t}^{l} \psi_{t+1}\left(u_{t}^{l}, \beta_{t}^{l}\right)= \\
& =\mathrm{E}_{t}^{u} \Psi_{t+1}\left(u_{t}^{l}, \beta_{t}^{l}\right)-\mathrm{E}_{t}^{l} \Psi_{t+1}\left(u_{t}^{l}, \beta_{t}^{l}\right)+\mathrm{E}_{t}^{l} \Psi_{t+1}\left(u_{t}^{l}, \beta_{t}^{l}\right)-\mathrm{E}_{t}^{l} \psi_{t+1}\left(u_{t}^{l}, \beta_{t}^{l}\right)= \\
& =\delta_{t}\left(\beta_{t}^{l}\right)+\sum_{\beta_{t+1}^{l} \in A_{t+1}^{l}\left(\beta_{t}^{l}\right)} \epsilon_{t+1}\left(\beta_{t+1}^{l}\right) \cdot q_{t+1}^{l}\left(\beta_{t+1}^{l} \mid \beta_{t}^{l}\right)=: \Delta_{t}\left(\beta_{t}^{l}\right) .
\end{aligned}
$$

According to the above relation, $\delta_{t}\left(\beta_{t}^{l}\right)$ is an upper bound for the error increment from period $t+1$ to period $t$ conditioned on $\beta_{t}^{l}$. Note that

$$
\epsilon_{t}\left(\beta_{t}^{l}\right)=\delta_{t}\left(\beta_{t}^{l}\right)+\sum_{\beta_{t+1}^{l} \in A_{t+1}^{l}\left(\beta_{t}^{l}\right)} \epsilon_{t+1}\left(\beta_{t+1}^{l}\right) \cdot q_{t+1}^{l}\left(\beta_{t+1}^{l} \mid \beta_{t}^{l}\right)=\Delta_{t}\left(\beta_{t}^{l}\right)
$$

holds in case $u_{t}^{l}$ is a minimizer of $\left[\rho_{t}+\mathrm{E}_{t}^{u} \Psi_{t+1}\right]\left(\cdot, \beta_{t}^{l}\right)$. This implies that the error increment is due to integration of $\Psi_{t+1}\left(u_{t}^{l}, \beta_{t}^{l}\right)$. If, additionaly, $\Psi_{t+1}\left(u_{t}^{l}, \beta_{t}^{l}\right)$ is bilinear, then $\delta_{t}\left(\beta_{t}^{l}\right)=0$ and

$$
\epsilon_{t}\left(\beta_{t}^{l}\right)=\sum_{\beta_{t+1}^{l} \in A_{t+1}^{l}\left(\beta_{t}^{l}\right)} \epsilon_{t+1}\left(\beta_{t+1}^{l}\right) \cdot q_{t+1}^{l}\left(\beta_{t+1}^{l} \mid \beta_{t}^{l}\right)=\Delta_{t}\left(\beta_{t}^{l}\right) .
$$

The above relations are illustrated on a 3-stage funding problem for the ease of understanding.

The optimal value of the lower approximation is 5906.92 and of the upper approximation is 6694.96 , the accuracy is $11.77 \%$ (see table 1 ). Three refinements have been performed which have decreased the relative error to $5.81 \%$. It is observed that the upper bound remains unchanged with respect to these refinements. This is due to fact that in the underlying funding problem the inner approximation $\Psi_{t}(\cdot)$ of the value function $\phi_{t}(\cdot)$ has been bilinear over $t=2,1,0$, for which case the nested integration and minimization of $\Psi_{t}(\cdot)$ is exact, and, hence, the upper bound and the corresponding minimizers remain unchanged. 


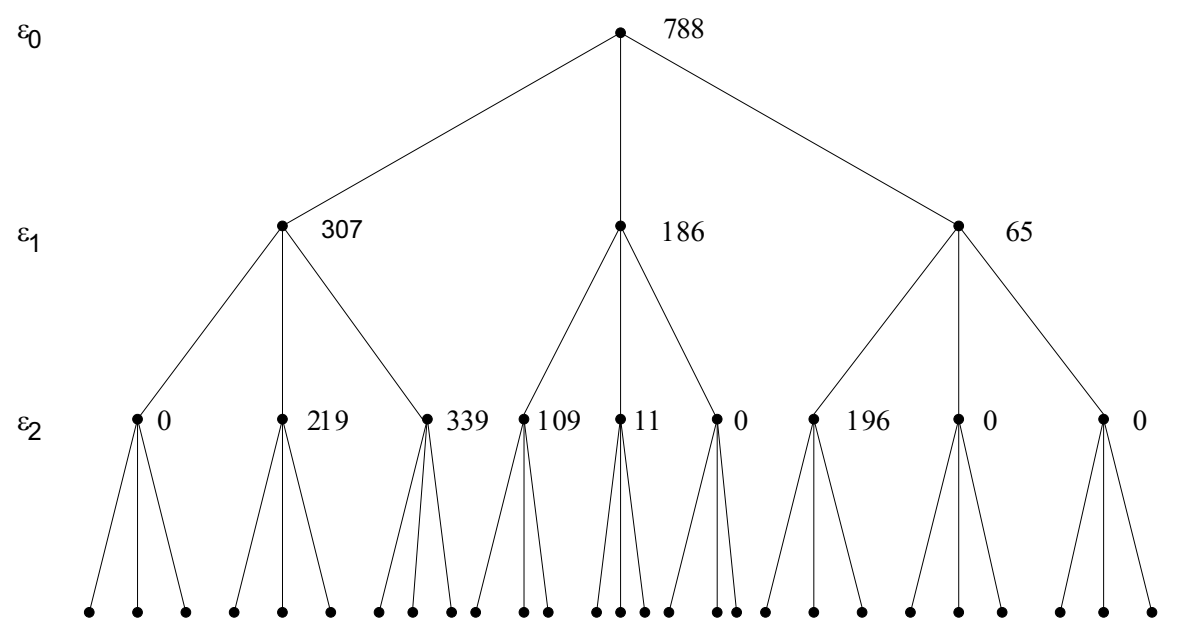

Figure 12: Evolvement of the error $\epsilon_{t}\left(\beta_{t}^{l}\right)$ backwards in time

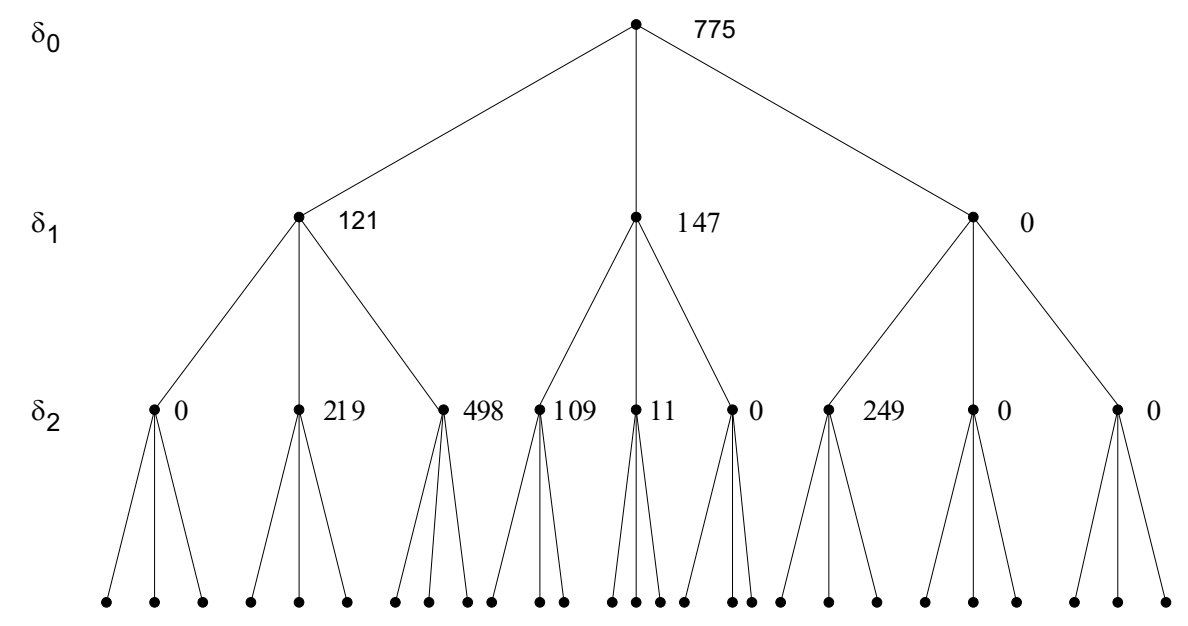

Figure 13: Error increments $\delta_{t-1}\left(\beta_{t-1}^{l}\right)$ at the nodes (backwards in time)

The evolvement of $\epsilon_{t}\left(\beta_{t}^{l}\right), \delta_{t}\left(\beta_{t}^{l}\right)$ and $\Delta_{t}\left(\beta_{t}^{l}\right)$ is illustrated in figures 12, 13, and 14. Nodes in 12 at which $\epsilon_{t}=0$ indicate that inner and outer approximation of the value functions coincide and are bilinear. Nodes in 13 at which $\delta_{t}=0$ indicate that the inner approximation is bilinear. Nodes at which $\epsilon_{t}=\Delta_{t}$ indicate that the error increment from stage $t+1$ to $t$ is due to inaccurate integration of the inner approximation, with no impact on the minimizer of the inner and outer approximation. This way, critical nodes may be assessed beyond which the corresponding coverage process should be refined. Given a node of $A^{l}$ conditioned on which the partitions are refined, one is faced with the two-stage situation. Theoretically, the various refinement schemes that have been developed by Kall and Stoyan 1982 [33], Birge and Wets 1986 [4], Frauendorfer and Kall 1988 [25], Kall, Ruszczyński and Frauendorfer 1988 [32], Edirisinghe and Ziemba 1994/1996 


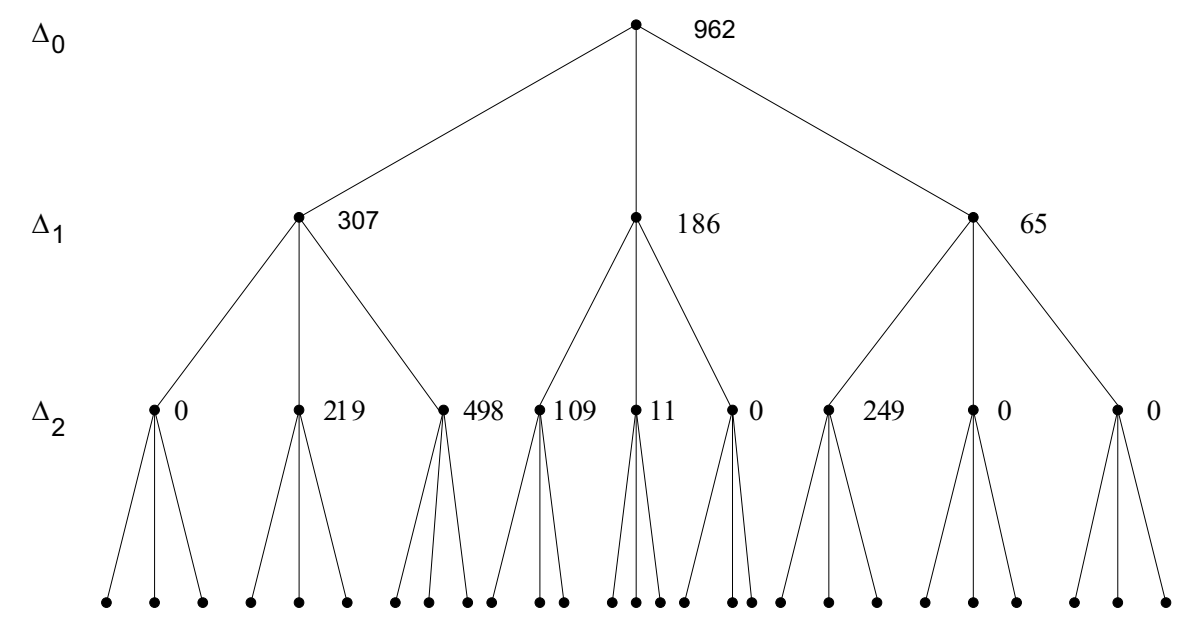

Figure 14: Error bounds $\Delta_{t-1}\left(\beta_{t-1}^{l}\right)$ of 3-stage scenario tree of lower approximation

[18], [17], [19], are applicable. However, note that contrary to the two-stage stochastic programs, the recourse function of the nested two-stage formulation within the multistage problem is only approximately available by the implicitely given inner and outer approximation. This fact certainly has to be taken into account and requires further investigations.

\section{Conclusions}

This work contributes to the solvability of stochastic multistage linear programs, which suffers from the nested optimization and multidimensional integration of implicitely given value functions. In the convex case, which holds if the conditional probability distributions depend linearly on the past and remain unaffected by the desicions taken, structural properties help to overcome numerical difficulties to a certain extent. In particular, applying the barycentric approximation technique yields distinguished scenario trees. The solution of the associated deterministic equivalent programs provides lower and upper bounds of the minimal expected costs given the entire planning horizon $[0, T]$. The approximate policies refer to different scenario trees and, hence, may differ so that the inaccuracies beyond $t \geq 1$ cannot be assessed immediately. The inner and outer approximation of the value function are implicitely given and may only be compared as long they refer to the same history. Due to the convexity of the value function with respect to the decisions, it has been realized that the outer approximation ensures the minorization of the minimum values subject to the stages $t=0,1, \ldots, T$. This has been the basis to focus on the corresponding scenario tree $A^{l}$, and, then to evaluate the upper bound with respect to each node of that tree. This way the 
error can be assessed with respect to any history within $A^{l}$. It has been observed that the error caused by integration of the inner approximation is mainly responsible for the error increments backwards in time. For determining the critical nodes beyond which the approximation should be refined, relations have been derived which characterize the total error at stages $t, t+1$ and the integration error that arises from $t+1$ to $t$. Given a history (i.e., a node) of $A^{l}$ conditioned on which the coverage process has to be refined, one is faced with the two-stage situation. However, for applying the various refinement schemes that have been developed for stochastic two-stage programs, it has to be taken into account that the recourse functions of the nested two-stage formulation within the multistage setting are only approximately available by the implicitely given value functions of the surrogate problems. This certainly opens further research activities which hopefully help increase the solvability and, hence, the applicability of stochastic multistage programs.

\section{References}

[1] Bertocchi, M., Dupačová, J., and Moriggia, V. Postoptimality for scenario based financial planning models with an application to bond portfolio management. In Worldwide Asset and Liablility Management, W. T. Ziemba and J. M. Mulvey, Eds. Cambridge University Press, 1997.

[2] Bertsekas, D. P. Dynamic Programming and Optimal Control, vol. 1. Athena Scientific, 1995.

[3] Bertsekas, D. P. Dynamic Programming and Optimal Control, vol. 2. Athena Scientific, 1995.

[4] Birge, J., And Wets, R. J.-B. Designing approximation schemes for stochastic optimization problems, in particular for stochastic programs with recourse. Mathematical Programming Study 27 (1986), 54-102.

[5] Birge, J. R. Decomposition and partitioning methods for multistage stochastic linear programs. Operations Research 33, 5 (1985), 989-1007.

[6] Birge, J. R. Current trends in stochastic programming computation and applications. Working paper, Department of Industrial and Operations Engineering, The University of Michigan, Ann Arbor, August 1995.

[7] Birge, J. R., And Rosa, C. H. Parallel decomposition of large-scale stochastic nonlinear programs. Working paper, Department of Industrial and Operations Engineering, The University of Michigan, Ann Arbor, 1994. 
[8] Cariño, D. R., And Kent, T. Multistage planning for asset allocation. In Worldwide Asset and Liablility Management, W. T. Ziemba and J. M. Mulvey, Eds. Cambridge University Press, 1997.

[9] Cariño, D. R., Kent, T., Myers, D. H., Stacy, C., Sylvanus, M., Turner, A. L., Watanabe, K., And Ziemba, W. T. The RussellYasuada kasai finanical planning model: An asset/liability model for a japanese insurance company using multistage stochastic programming. In Worldwide Asset and Liablility Management, W. T. Ziemba and J. M. Mulvey, Eds. Cambridge University Press, 1997.

[10] Cariño, D. R., Myers, D. H., and Ziemba, W. T. Concepts, technical issues, and uses of the Russel-Yasuda kasai financial planning model. Working paper, Frank Russell Company, May 1995.

[11] Consigli, G., And Dempster, M. A. H. Dynamic stochastic programming for asset-liability management. In Worldwide Asset and Liablility Management, W. T. Ziemba and J. M. Mulvey, Eds. Cambridge University Press, 1997.

[12] Dahl, H., Meeraus, A., and Zenios, S. A. Some financial optimization models: I. risk management. In Financial Optimization, S. A. Zenios, Ed. Cambridge University Press, 1993, pp. 3-36.

[13] Dantzig, G. B., And Glynn, P. W. Parallel processors for planning under uncertainty. Annals of Operations Research 22 (1990), 1-21.

[14] Dantzig, G. B., And Infanger, G. Multi-stage stochastic linear programs for portfolio optimization. Annals of Operations Research 45 (1993), $59-76$.

[15] DupaČová, J. Stochastic programming models in banking. Working paper, International Institute for Applied Systems Analysis (IIASA), 1991.

[16] Dupačová, J. Portfolio optimization under uncertainty. submitted for Annals of Operations Research (1992).

[17] Edirisinghe, N. C. P., And ZiembA, W. T. Bounding the expectation of a saddle function with application to stochastic programming. Mathematics of Operations Research 19, 2 (1994), 314-340.

[18] Edirisinghe, N. C. P., And Ziemba, W. T. Bounds for two-stage stochastic programs with fixed recourse. Mathematics of Operations Research 19, 2 (1994), 292-313. 
[19] Edirisinghe, N. C. P., and Ziemba, W. T. Implementing boundsbased approximations in convex-concave two-stage stochastic programming. Mathematical Programming 75, 2 (1996), 295-326.

[20] Ermoliev, Y., And Wets, R. J.-B., Eds. Numerical Techniques for Stochastic Optimization. Springer-Verlag, Berlin, 1988.

[21] Frauendorfer, K. Stochastic Two-Stage Programming. Lecture Notes in Economics and Mathematical Systems 392. Springer-Verlag, Berlin, 1992.

[22] Frauendorfer, K. Multistage stochastic programming: Error analysis for the convex case. Zeitschrift für Operations Research 39 (1994), 93-122.

[23] Frauendorfer, K. Barycentric scenario trees in convex multistage stochastic programming. Mathematical Programming 75, 2 (1996), 277-294.

[24] Frauendorfer, K. Stochastic multistage programming in financial decision making. Zeitschrift für Angewandte Mathematik und Mechanik 76 (1996), $21-24$.

[25] Frauendorfer, K., And Kall, P. A solution method for SLP recourse problems with arbitrary multivariate distribution - the independent case. Problems of Control and Information Theory 17 (1988), 177-205.

[26] Frauendorfer, K., And Schürle, M. Barycentric approximation of stochastic interest rate processes. In Worldwide Asset and Liablility Management, W. T. Ziemba and J. M. Mulvey, Eds. Cambridge University Press, 1997.

[27] Gassmann, H. MSLIP: A computer code for the multistage stochastic linear programming problem. Mathematical Programming (1990), 407-423.

[28] Golub, B., Holmer, M., McKendall, R., Pohlman, L., and Zenios, S. A. A stochastic programming model for money management. European Journal of Operational Research (1995), 282-296.

[29] Hiller, R. S., And Eckstein, J. Stochastic dedication: Designing fixed income portfolios using massively parallel benders decomposition. Management Science 39, 11 (1994), 1422-1438.

[30] Infanger, G. Monte carlo (importance) sampling within a benders decomposition algorithm for stochastic linear programs. Annals of Operations Research 39 (1992), 69-95.

[31] Infanger, G. Planning under Uncertainty, Solving Large-Scale Stochastic Linear Programs. The Scientific Press Series, Danvers, Massachusetts, 1994. 
[32] Kall, P., Ruszczyński, A., and Frauendorfer, K. Approximation techniques in stochastic programming. In Numerical Techniques for Stochastic Optimization, Y. Ermoliev and R. J.-B. Wets, Eds. Springer-Verlag, 1988, pp. 33-64.

[33] Kall, P., And Stoyan, D. Solving stochastic programming problems with recourse including error bounds. Mathematische Operationsforschung und Statistik Series Optimization 13 (1982), 431-447.

[34] Kall, P., And Wallace, S. W. Stochastic Programming. Wiley and Sons Ltd., Chichester, 1994.

[35] Klanassen, P. Financial asset-pricing theory and stochastic programming models for asset/liability management: a synthesis. In Worldwide Asset and Liablility Management, W. T. Ziemba and J. M. Mulvey, Eds. Cambridge University Press, 1997.

[36] Kusy, M. I., And Ziemba, W. T. A bank asset and liability management model. Operations Research 34 (1986), 356-376.

[37] Mulvey, J. M. Multi-stage financial planning. In Operations Research Models in Quantitative Finance, R. L. D'Ecclesia and S. A. Zenios, Eds. Physica-Verlag, 1994, pp. 18-35.

[38] Mulvey, J. M., And Ruszczyński, A. A new scenario decomposition method for large scale stochastic optimization. Operations Research 43 (1995), $477-453$.

[39] Mulvey, J. M., And Thorlacius, E. The tower perrin global capital market scenario generation system: Cap-link. In Worldwide Asset and Liablility Management, W. T. Ziemba and J. M. Mulvey, Eds. Cambridge University Press, 1997.

[40] Mulvey, J. M., Vanderbei, R., And Zenios, S. Robust optimization of large scale systems. Operations Research 43, 2 (1995), 264-281.

[41] Mulvey, J. M., And Vladimirou, H. Stochastic network programming for financial planning problems. Management Science 38 (1992), 1642-1664.

[42] Mulvey, J. M., And Ziemba, W. T. Asset and liability modeling: discussion of issues. In Worldwide Asset and Liablility Management, W. T. Ziemba and J. M. Mulvey, Eds. Cambridge University Press, 1997.

[43] Nielsen, S. S., And Zenios, S. A. A stochastic programming model for funding single premium deferred annuities. Mathematical Programming 75, 2 (1996), 177-200. 
[44] Robinson, S. M. Extended scenario analysis. Annals of Operations Research 31 (1991), 385-398.

[45] Rockafellar, R. T., And Wets, R. J.-B. Nonanticipativity and L ${ }^{1}$ martingales in stochastic optimization problems. Mathematical Programming 6 (1976), 170-187.

[46] Rockafellar, R. T., And Wets, R. J.-B. The optimal recourse problem in discrete time: $\mathrm{L}^{1}$-multipliers for inequality constraints. SIAM Journal on Control and Optimization 16, 1 (1978), 16-36.

[47] Rockafellar, R. T., And Wets, R. J.-B. Scenarios and policy aggregation in optimization under uncertainty. Mathematics of Operations Research 16, 1 (1991), 119-147.

[48] Ruszczyński, A. Interior point methods in stochastic programming. Working paper, International Institute for Applied Systems Analysis, Laxenburg, Februar 1993.

[49] Ruszczyński, A. Parallel decomposition of multistage stochastic programming problems. Mathematical Programming 58, 2 (1993), 201-228.

[50] Vassiadou-Zeniou, C., And Zenios, S. A. Robust optimization models for managing callable bond portfolios. Interfaces 24 (1994), 29-49.

[51] Wets, R. J.-B. The aggregation principle in scenario analysis and stochastic optimization. In Algorithms and Model Formulations in Mathematical Programming, S. W. Wallace, Ed. Springer, Berlin, Heidelberg, 1989, pp. 91113.

[52] Zenios, S. A. Massively parallels computations for financial planning under uncertainty. In Very Large Scale Computing in the 21-st Century, J. Mesirov, Ed. SIAM, Philadelphia, 1991.

[53] Zenios, S. A. Asset/liability management under uncertainty: The case of mortgage-backed securities. Technical report, Department of Decision Sciences, The Wharton School, University of Pennsylvania, Philadelphia, 1992.

[54] Zenios, S. A., Ed. Financial Optimization. Cambridge University Press, 1992.

[55] Zenios, S. A., And Ziemba, W. T. Financial modeling. Management Science 38 (1992).

[56] Ziemba, W. T., And Mulvey, J. M., Eds. Worldwide Asset and Liability Management. Cambridge University Press, 1997. 
[57] Ziemba, W. T., And Vickson, R. G., Eds. Stochastic Optimization Models in Finance. Academic Press, New York, 1975. 